\title{
Pressure-Induced Phase Transformation in $\beta$-Eucryptite: an X-Ray Diffraction and Density Functional Theory Study
}

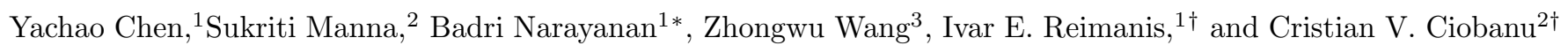 \\ ${ }^{1}$ Department of Metallurgical and Materials Engineering, \\ Colorado School of Mines, Golden, Colorado 80401, USA \\ ${ }^{2}$ Department of Mechanical Engineering, Colorado School of Mines, Golden, Colorado 80401, USA and \\ ${ }^{3}$ Cornell High Energy Synchrotron Source, Cornell University, Ithaca, New York 14853, USA
}

\begin{abstract}
Certain alumino-silicates display exotic properties enabled by their framework structure made of corner-sharing tetrahedral rigid units. Using in situ diamond-anvil cell x-ray diffraction (XRD), we study the pressure-induced transformation of $\beta$ eucryptite, a prototypical alumino-silicate that undergoes a phase transformation at moderate pressures. The atomic structure and symmetry group of the new pressure-stabilized phase has not yet been reported. Based on density functional theory studies and Rietveld analysis of XRD patterns, we find that the new phase belongs to the Pna2 $2_{1}$ space group and report its atomic structure. Furthermore, we discover two other possible pressure-stabilized polymorphs, $\mathrm{P} 1 \mathrm{c} 1$ and $\mathrm{Pca} 22_{1}$.
\end{abstract}

From their ubiquity in silica phases found in Earth's mantle ${ }^{1-3}$ to their importance for synthesizing ceramics with extraordinary electronic ${ }^{4}$ or mechanical properties, ${ }^{5,6}$ pressure-induced phase transformations in silicates provide fundamental insights into structure-property relations as well as ways to control these relations so as to enable a variety of engineering applications. Such applications range from transformation toughening in ceramics ${ }^{7}$ to cathode materials for Li-ion batteries. ${ }^{89}$ Amongst silicates, $\mathrm{LiAlSiO}_{4}$ displays a wide variety of pressure-induced transformations. Their structure consists of tetrahedra centered at $\mathrm{Si}$ or $\mathrm{Al}$, connected in an anisotropic framework that weakly binds lithium ions in the voids surrounded by the tetrahedra. ${ }^{11}$ This framework underlies a whole host of exotic phenomena and properties, such as negative coefficient of thermal expansion, ${ }^{10-15}$ negative compressibility, and one-dimensional ionic conduction. ${ }^{13,16-19}$ In addition, $\beta$-eucryptite (a $\mathrm{LiAlSiO}_{4}$ polymorph) exhibits a pressure-induced phase transformation at pressures that are low enough to be exploited technologically. ${ }^{20,21}$

The crystal structure of $\beta$-eucryptite (space group $\mathrm{P} 6_{4} 22$ or $\mathrm{P} 6_{2} 22$ ) can be described as a stuffed derivative of

\footnotetext{
* Present address: Nanoscience and Technology Division, Argonne National Laboratory, 9700 S. Cass Avenue, Argonne, IL 60439

† Corresponding authors. Email: reimanis@mines.edu, cciobanu@mines.edu
} 
$\beta$-quartz. ${ }^{10,12-14}$ For this structure, Morosin et al. ${ }^{15}$ reported a phase transformation occurring at pressures as low as $\sim 0.8 \mathrm{GPa}$. Zhang et al. ${ }^{22,23}$ also found a new polymorph (dubbed the $\epsilon$ phase) around 1 GPa, reporting that the transformation from $\beta$ to $\epsilon$ was reversible; amorphization was found to occur at pressures above 5 GPa. ${ }^{22}$ More recent indentation and Raman spectroscopy experiments revealed that the critical pressure for the forward transformation $(\beta$ to $\epsilon)$ is higher than that for the reverse one, ${ }^{20}$ suggesting that it may be possible to produce metastable eucryptite phases under ambient conditions.

While there is no doubt that a phase transformation from $\beta$-eucryptite to another crystalline phase exists at low pressures, the atomic structure of that phase, $\epsilon$, remains unknown. To date, the crystal system, lattice constants, and Miller indices associated with the $\epsilon$ phase diffraction peaks reported by Zhang et $a .^{22}$ are in doubt as they have not been reproduced by other groups. Here, we have carried out x-ray diffraction (XRD) experiments and density functional theory (DFT) calculations in order to elucidate the atomic structure, symmetry group, and the XRD indexing of the $\epsilon$ phase. We have found that there are several polymorphs that become more stable than the $\beta$ phase upon increasing pressure. From these, we have determined that the best match for the experimental XRD patterns is achieved by an orthorhombic Pna $2_{1}$ structure. Furthermore, we have found that the presence of Mg as a dopant leads to the observation of two phases that coexist past the transition pressure, and identified their atomic structures as well. This finding indicates a subtle competition between polymorphs under pressure, and suggests a way to modify the number and type of coexisting phases that could be used in transformation toughening.

In our experiments, $\beta$-eucryptite powders have been synthesized through a sol-gel route. ${ }^{24-26}$ We have doped some of the samples with $\mathrm{Mg}$, substituting for lithium up to $0.3 \mathrm{~mol} \%$. XRD measurements under pressure have been performed in situ at room temperature in a diamond anvil cell (DAC), from ambient pressure to 5 GPa; more details are given in Supplemental Material (SM). The pressure $p$ is derived from changes in the ruby fluorescence ${ }^{27}$ via $p(\mathrm{GPa})=0.274 \Delta \Lambda$, where $\Delta \Lambda(\AA)$ is the difference between the ruby wavelength being detected and that at ambient pressure, $6942.1 \AA .^{28}$ As evidenced by the lack of band splitting in ruby fluorescence, shear was absent ${ }^{29,30}$ in the sample, so all experiments have been carried out in hydrostatic conditions.

In situ DAC XRD experiments were conducted using an angle dispersive synchrotron source at Cornell High Energy Synchrotron Source (CHESS). ${ }^{31}$ Incident X-rays were converted by a double-bouncing monochromator at a wavelength of $\lambda=0.48596 \AA$. Our data [Fig. 1(a)] shows that at ambient pressure only the $\beta$ phase is present, which has a dominant peak [Fig. 1(b)] corresponding to the (202) planes. Above a threshold pressure, this peak changes shape as shown in Fig. 1(c): new, smaller peaks appear at that pressure, and are marked by black dots on the $2.47 \mathrm{GPa}$ curve in 

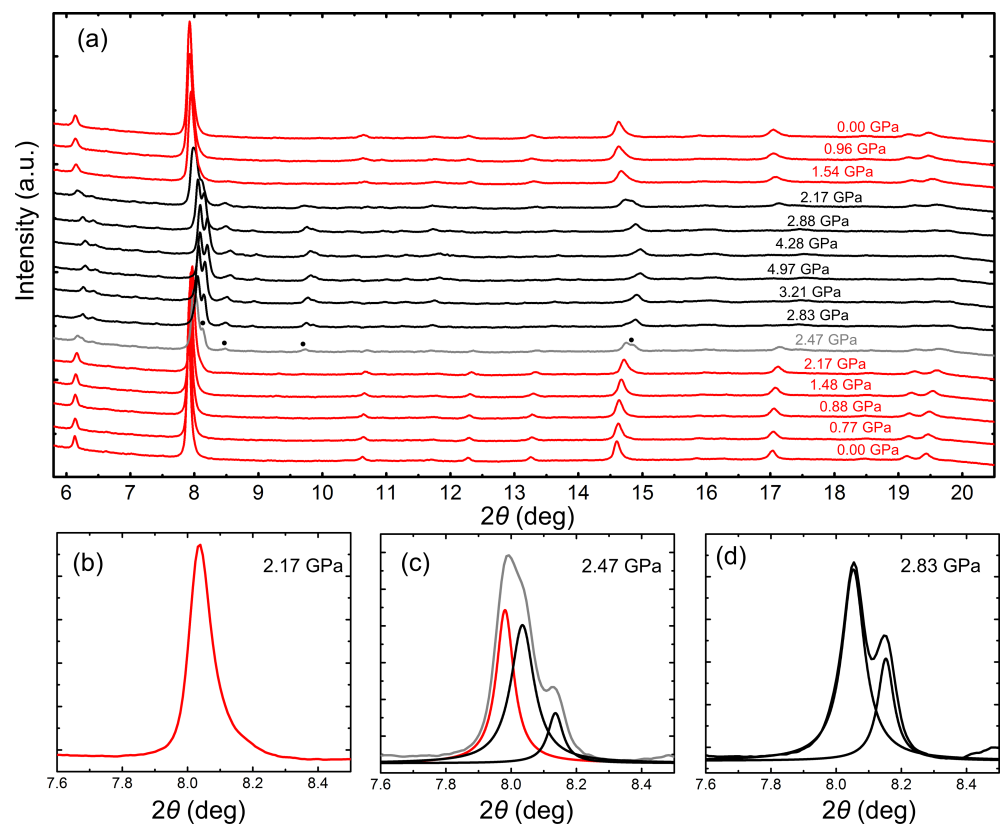

FIG. 1: XRD patterns showing the pressure-induced phase transformation in $\beta$-eucryptite. (a) Variation of the pattern with pressure in a loading-unloading cycle. The patterns that contain the new phase(s) are shown in black, while the $\beta$-phase is red. (b)-(d) Detail views of the main peak at three pressures around the transition, showing the main peaks (b) before, (c) during, and (d) after the transition.

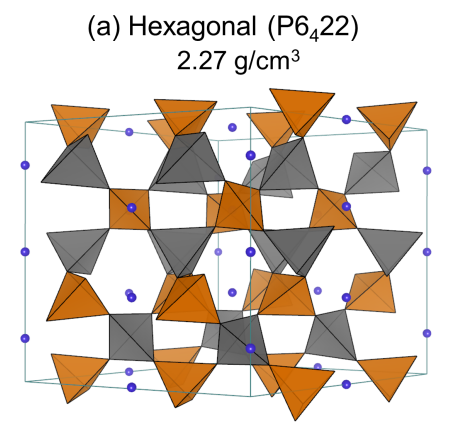

(c) Orthorhombic $\left(\mathrm{Pca}_{1}\right)$

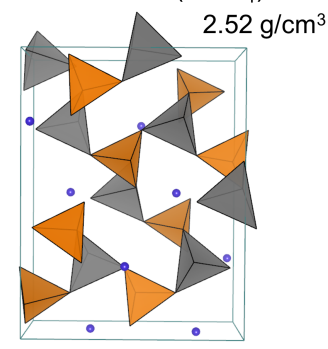

(b) Monoclinic (P1c1)

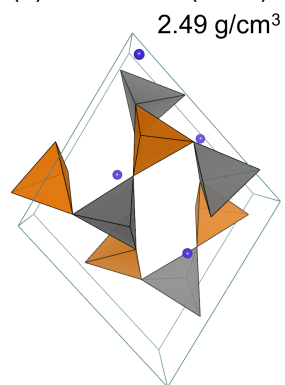

(d) Orthorhombic $\left(\mathrm{Pna2}_{1}\right)$

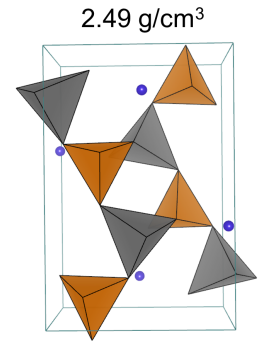

FIG. 2: Crystal structures of (a) $\beta$-eucryptite and (b)-(d) three denser polymorphs, and their densities at zero pressure. Al-(Si-)centered tetrahedra are shown in gray $(\tan )$, Li atoms are purple spheres. 


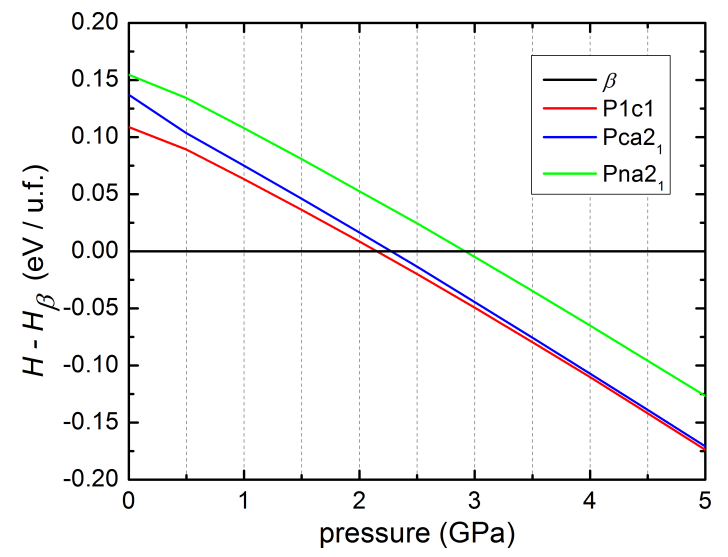

FIG. 3: Pressure-dependent enthalpy of $\mathrm{LiAlSiO}_{4}$ polymorphs, with respect to the $\beta$-phase.
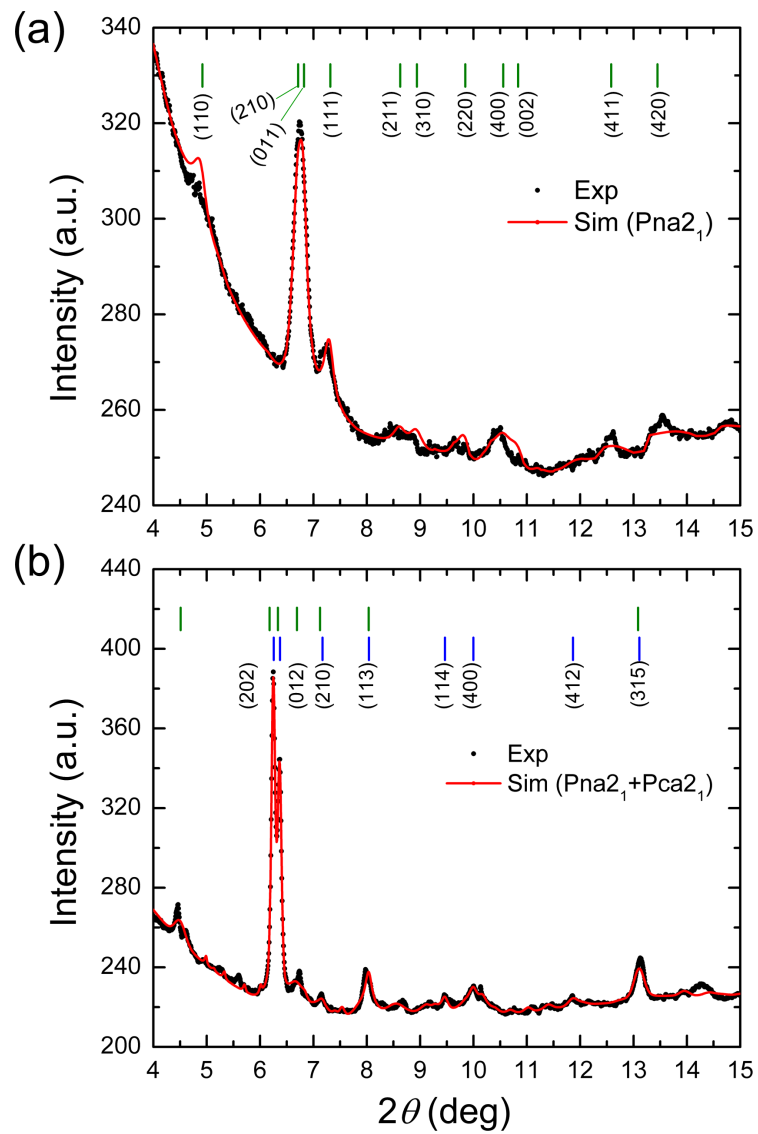

FIG. 4: (a) XRD pattern for the pure $\beta$-phase at $5 \mathrm{GPa}$, compared with the optimized simulated pattern for the Pna2 phase; the other polymorphs have significant deviations from the experimental pattern. (b) XRD pattern of the Mg-doped $\beta$-eucryptite at $5 \mathrm{GPa}$, compared with simulations of optimized mixture of Pna $2_{1}$ and Pca2 $2_{1}$. For clarity, only the Miller indices corresponding to $\mathrm{Pca} 2_{1}$ peaks are shown in panel (b). 
Fig. 1(a). By fitting the complex peak shape at 2.47GPa [Fig. 1(c)] with Lorentzian functions, we identify three peak locations - one corresponding to $\beta$, and the other two corresponding to the new phase(s). A small increase in pressure past 2.47 GPa leads to the disappearance of the main $\beta$ peak, while the intensity of the other two peaks increases [Fig. 1(d)]. Thus, for the Mg-doped samples analyzed, the transition occurs between 2.47GPa and 2.83GPa, with the $\beta$ phase absent past 2.83GPa; pure samples exhibit somewhat smaller transition pressures.

We now focus on searching for crystalline phases that can become more stable than $\beta$ upon pressure loading, and hence could be the product of the pressure-induced phase transformation of $\beta$. To this end, we have analyzed over thirty structures made of corner-sharing $\mathrm{AlO}_{4}$ and $\mathrm{SiO}_{4}$ tetrahedra such that any Al-centered tetrahedron is surrounded by four Si-centered tetrahedra, and vice-versa. These structures have been obtained in two ways: (a) by creating Li-stuffed derivatives of known $\mathrm{SiO}_{2}$ structures, similar to the way in which $\beta$-eucryptite is related to $\beta$-quartz; and (b) by converting the alkaline atom $\mathrm{M}(\mathrm{M}=\mathrm{Li}, \mathrm{Na}, \mathrm{K})$ of known $\mathrm{MAlSiO}_{4}$ and $\mathrm{MAlGeO}_{4}$ structures $^{32}$ into Li, and Ge into Si when necessary. All structures have been relaxed using DFT in the generalized gradient approximation (GGA), ${ }^{33}$ for each pressure from 0 to $5 \mathrm{GPa}$ in increments of $0.5 \mathrm{GPa}$. After eliminating duplicates, the crystals that we identified as having higher density than $\beta$ and low heat of formation are shown in Fig. 2, with the space group and density at zero pressure identified; $\beta$ is included as reference. The higher-density phases also have lower symmetry, as expected from the symmetry hierarchy of crystal systems. ${ }^{37}$ Two of the three new phases are orthorhombic, with space groups no. 33 (Pna2 $\left.2_{1}\right)$ and 29 (Pca2 $\left.2_{1}\right)$; the remaining phase is monoclinic (group no. 7, P1c1).

Next, we discuss the relative stability of these phases with respect to $\beta$. In Fig. 3, we plot the difference between the enthalpy of each candidate phase and that of $\beta$, per unit formula. Interestingly, past a certain pressure each of these phases becomes more stable than $\beta$ eucryptite (Fig. 3); for these pressure-stabilized phases, the lattice parameters are given in Table I, and the atomic positions are given in SM. Furthermore, all threshold pressures fall between $2 \mathrm{GPa}$ and $3 \mathrm{GPa}$ (Fig. 3), in good agreement with the experimental assessment of the range of pressures required for transition (Fig. 1). We cannot expect a more quantitative agreement because of the uncertainties related to the indirect pressure measurements in the DAC, and of the approximations made in the DFT calculations (for example, the enthalpies are computed at $0 \mathrm{~K}$ and not at room temperature). In order to identify more precisely the phases to which $\beta$ transitions upon loading, we examine the XRD patterns at high-pressures.

We have performed Rietveld analysis of the XRD data using MAUD software, ${ }^{38}$ designed to refine the background, structural (atomic coordinates, occupancies, lattice parameters and angles), and microstructural (particle size, lattice 
strain, residual stress, texture, etc) parameters via a least-squares method. ${ }^{39}$ As input, we have supplied the XRD pattern and a candidate structure with its atomic coordinates and symmetry group. The optimization proceeds with fitting the background and the peak shape; the shape of the peaks was assumed to be of asymmetric, pseudo-Voigt form so it can fit crystal size and strain broadening of the experimental profiles. ${ }^{40}$ Without refining the fractional atomic coordinates and thermal vibration parameters, the following parameters have been optimized: background coefficients, scale, lattice parameters, zero-shift error, and peak shape parameters. The procedure yields a simulated optimized spectrum for comparison with the experimental data. We have carried out this procedure for each of the candidate structures [Fig. 2(b)-(d)]. The best fit for the XRD pattern of the pure, undoped sample is the Pna2 1 structure, shown in Fig. 4(a) for an XRD pattern at $\sim 5 \mathrm{GPa}$.

\begin{tabular}{l|cccccc}
\hline \hline Phase & $a(\AA)$ & $b(\AA)$ & $c(\AA)$ & $\alpha\left(^{\circ}\right)$ & $\beta\left(^{\circ}\right)$ & $\gamma\left(^{\circ}\right)$ \\
\hline$\beta$ & 10.575 & 10.575 & 11.391 & 90.000 & 90.000 & 120.000 \\
P1c1 & 8.200 & 8.288 & 5.147 & 90.000 & 90.000 & 106.290 \\
Pca2 $_{1}$ & 10.079 & 5.033 & 13.114 & 90.000 & 90.000 & 90.000 \\
Pna2 $_{1}$ & 10.082 & 6.673 & 4.990 & 90.000 & 90.000 & 90.000 \\
\hline
\end{tabular}

TABLE I: Lattice parameters and angles for the crystal structures shown in Fig. 2 computed at zero pressure using GGA. The dependence on pressure is included in SM.

It is worthwhile to compare this result with the previous reports on the phase transformations of $\beta$ eucryptite. Morosin et al. observed a phase transformation around $0.8 \mathrm{GPa}$, and interpreted it as a hexagonal phase with lattice constants commensurate with half of those of $\beta .{ }^{15}$ The structure of our monoclinic P1c1 phase is stabilized at the lowest pressure (Fig. 3) and is somewhat close to being hexagonal ( $a$ and $c$ are within $1.1 \%$ of each other, and the angle between them $\sim 12 \%$ away from $120^{\circ}$ ), so it is reasonable to infer that this phase may actually be the one found by Morosin et al. ${ }^{41}$ Zhang et al. reported a reversible transformation around $1.5 \mathrm{GPa}$ to $\epsilon$ eucryptite: ${ }^{22}$ although these authors conclude that the $\epsilon$ phase is orthorhombic, its space group and the atomic structure have not been reported so far. Our data also indicates that $\epsilon$ eucryptite is orthorhombic, but with different lattice constants; in addition, we report here the space group (no. 33, Pna2 ${ }_{1}$ ) and the atomic structure [Fig. 2(e) and SM]. The previous indexing ${ }^{22}$ is not consistent with the reflection conditions for most orthorhombic groups. ${ }^{42}$ On the other hand, the planes that we have found in our XRD pattern [indexed in Fig. 4(a)] obey the reflection conditions for group no. 33, and are largely the same as those encountered in other materials with the space group Pna $2_{1} \cdot{ }^{43,44}$

We have also analysed XRD data of $0.3 \mathrm{~m} \% \mathrm{Mg}$ doped samples such as those in Fig. 1, and have found that no single 
phase results in a satisfactory fit to the XRD data. Therefore, we are led to assume that in the presence of $\mathrm{Mg}$, there can be two or more phases present at high pressures. We have analyzed phase mixtures using MAUD for all possible combinations of the phases shown in Fig. 2(b)-(d). The best fit for the XRD pattern of the Mg-doped eucryptite at $5 \mathrm{GPa}$ [shown in Fig. 4(b)] is obtained from a mixture of $45.3 \%$ (by volume) Pna $2_{1}$ and $54.7 \%$ Pca $2_{1}$. It is rather intriguing that the presence of $\mathrm{Mg}$ in very small amounts facilitates the occurrence of more than one crystalline phase upon pressure loading -which may be due to entropic (mixing) or kinetic factors.

In summary, we have used XRD measurements and DFT calculations to elucidate the structure of the pressurestabilized $\epsilon$ phase of eucryptite. While this answers a long-standing question, deeper investigations are necessary to understand how to control the presence of different polymorphs at low and moderate pressures. The reversible nature $\beta \rightarrow \epsilon$ transition, coupled with moderate values of the transition pressure (2-3 GPa) and of volume change $(\sim 9 \%)$, make it suitable for transformation toughening of ceramic composites for various applications. Knowledge of the $\epsilon$ phase can enable a better control over the design of such composites (e.g., optimizing the particle size, and distribution of $\beta$-eucryptite particles to be employed in a suitable matrix). Furthermore, we have found that the presence of small amounts of dopants facilitates the coexistence of distinct polymorphs under pressure, which may lead to novel properties displayed by such phase mixtures.

Acknowledgments. We gratefully acknowledge the support of U.S. Department of Energy's Office of Basic Energy Sciences through Grant No. DE-FG02-07ER46397 and that of the National Science Foundation for CHESS under Grant No. DMR-1332208.

${ }^{1}$ S. Zhang, S. Cottaar, T. Liu, S. Stackhouse, B. Militzer, Earth and Planetary Science Letters 434, 264 (2016).

2 T. Irifune, T. Shinmei, C. A. McCammon, N. Miyajima, D. C. Rubie, and D. J. Frost, Science 327, 193 (2010).

${ }^{3}$ K. J. Kingma, R. E. Cohen, R. J. Henley, and H. K. Mao, Nature 374, 243 (1995).

${ }^{4}$ D. Erradonea and F. J. Manjon, Progress in Materials Science 53, 711 (2008).

${ }^{5}$ A. Rosenflanz, M. Frey, B. Endres, T. Ansderson, E. Richards, C. Schardt, Nature 430, 761 (2004)

${ }^{6}$ H. P. Scott, Q. Williams, and E. Knittle, Phys. Rev. Lett. 88, 015506 (2002).

7 P. M. Kelly and L. R. F. Rose, Progress in Materials Science 47, 463 (2002).

8 J. T. Zhu, Z. Y. Tang, H. Q. Tang, Q. Xu, X. H. Zhang, J. Electrochemical Chemistry 761, 37 (2016).

9 T. Sarkar, M. Bharadwaj, U. V. Waghmare, P. Kumart, J. Phys. Chem. C 119, 9125 (2015).

${ }^{10}$ H. Xu, P. J. Heaney, and H. Bohm, Phys. Chem. Miner. 26, 633 (1999). 
${ }^{11}$ H. Xu, P. J. Heaney, D. M. Yates, R. B. Von Dreele, and M. A. Bourke, J. Mater. Res. 14, 3138 (1999).

12 V. Tscherry, H. Schulz, and F. Laves, Z. Kristallogr. 135, 175 (1972).

13 H. Schulz and V. Tscherry, Acta Crystallogr. B 28, 2174 (1972).

${ }^{14}$ F. Shin-ichi, S. Satoshi, S. Kaduhiro, and T. Hitoshi, Solid State Ionics 167, 325 (2004).

15 B. Morosin and P. S. Peercy, Phys. Lett. A 53, 147 (1975).

16 R. T. Johnson Jr., B. Morosin, M. L. Knotek, and R. M. Biefeld, Phys. Lett. A 54, 403 (1975).

17 U. V. Alpen, H. Schulz, G. H. Talat, and H. Bohm, Solid State Commun. 23, 911 (1977).

18 W. Press, B. Renker, H. Schulz, and H. Bohm, Phys. Rev. B 21, 1250 (1980).

19 B. Renker, H. Bernotat, G. Heger, N. Lehner, and W. Press, Solid State Ionics 9-10, Part2, 1341 (1983).

20 T. Jochum, I. E. Reimanis, M. J. Lance, and E. R. Fuller, J. Am. Ceram. Soc. 92, 857 (2009).

${ }^{21}$ S. Ramalingam, C. E. Packard, and I. E. Reimanis, J. Am. Ceram. Soc. 96, 1909 (2013).

22 J. Zhang, A. Celestian, J. B. Parise, H. Xu, and P. J. Heaney, Am. Mineral. 87, 566 (2002).

${ }^{23}$ J. Zhang, Y. Zhao, H. Xu, M. V. Zelinskas, L. Wang, Y. Wang, and T. Uchida, Chem. Mater. 17, 2817 (2005).

${ }^{24}$ D. Mazza, M. Lucco-Borlera, G. Busca, and A. Delmas- tro, J. Eur. Ceram. Soc. 11, 299 (1993).

25 D. Mazza and M. Lucco-Borlera, J. Eur. Ceram. Soc. 13, 61 (1994).

26 S. Ramalingam and I. E. Reimanis, J. Am. Ceram. Soc. 95, 2939 (2012).

27 H. K. Mao, J. Xu, and P. M. Bell, J. Geophys. Res. 91, 4673 (1986).

${ }^{28}$ H. Yamaoka, Y. Zekko, I. Jarrige, J.-F. Lin, N. Hiraoka, H. Ishii, K.-D. Tsuei, and J. Mizuki, J. App. Phys. 112, 124503 $(2012)$

29 M. Chai and J. M. Brown, Geophys. Res. Lett. 23, 3539 (1996).

${ }^{30}$ K. Syassen, High Pressure Res. 28, 75 (2008).

31 Z. Wang, O. Chen, C. Y. Cao, K. Finkelstein, D.-M. Smilgies, X. Lu, and W. A. Bassett, Rev. Sci. Instrum. 81, 093902 (2010).

32 https://www.materialsproject.org/

33 The DFT calculations were performed with $\mathrm{VASP}^{34}$ at the level of generalized gradient approximation using the PerdewBurke-Ernzenhof exchange-correlation functional. ${ }^{35}$ We have used projector-augmented pseudopotentials, ${ }^{36}$ a 500 eV planewave energy cutoff, and $3 \times 3 \times 3 k$-point grid for supercells with lattice parameters $\geq 10 \AA$ (primitive cells repeated accordingly). The atomic and lattice paramater relaxations were stopped when residual forces became smaller than 0.01 $\mathrm{eV} / \AA$.

${ }^{34}$ G. Kresse and J. Furthmüller, Comp. Mat. Sci. 6, 15 (1996); ibid, Phys. Rev. B 54, 11169 (1996).

35 J. P. Perdew, K. Burke, and M. Ernzerhof, Phys. Rev. Lett. 77, 3865 (1996).

${ }^{36}$ G. Kresse and D. Joubert, Phys. Rev. B 59, 1758 (1999). 
37 G. Burns and M. Glazer, Space Groups for Solid State Scientists, Academic Press, Third Ed. (2013).

${ }^{38}$ L. Lutterotti, MAUD, version 2.55 released April 30, 2015, http://www.ing.unitn.it/ luttero/maud

39 L. Lutterotti, P. Scardi, P. Maistrelli, J. Appl. Crystallogr. 25459 (1992).

${ }^{40}$ F. Sánchez-Bajo and F. L. Cumbrera, J. Appl. Cryst. 30, 427 (1997).

41 XRD patterns of the pressure-stabilized phase were not published in Ref. 15, so this hypothesis cannot be readily verified.

42 International Tables for Crystallography, Vol. A: Space Group Symmetry, Ed. T. Hahn, Fifth Ed., pp. 191-321, Springer (2005). Reflection conditions for group no. 33 are given at p. 225.

43 J. Barbier, N. Penin, A. Denoyer, and L. M. D. Cranswick, Solid State Sci. 7, 1055 (2005).

44 A. F. Goncharov, M. R. Manaa, J. M. Zaug, R. H. Gee, L. E. Fried, W. B. Montgomery, Phys. Rev. Lett. 94, 065505 (2005). 
